\title{
Heat Transfer Analysis of Viscous Incompressible Fluid by Combined Natural Convection and Radiation in an Open Cavity
}

\author{
M. Saleem, ${ }^{1}$ M. A. Hossain, ${ }^{2}$ Suvash C. Saha, ${ }^{3}$ and Y. T. Gu ${ }^{3}$ \\ ${ }^{1}$ Department of Electrical and Computer Engineering, Center for Advanced Studies in Engineering, 19 Atta Turk Avenue, G-5/1, \\ Islamabad 44000, Pakistan \\ ${ }^{2}$ University of Dhaka, Dhaka 1000, Bangladesh \\ ${ }^{3}$ School of Chemistry, Physics \& Mechanical Engineering, Queensland University of Technology, G.P.O. Box 2434, \\ Brisbane, QLD 4001, Australia \\ Correspondence should be addressed to Suvash C. Saha; s_c_saha@yahoo.com
}

Received 10 March 2014; Revised 24 May 2014; Accepted 3 June 2014; Published 25 June 2014

Academic Editor: Hang Xu

Copyright (C) 2014 M. Saleem et al. This is an open access article distributed under the Creative Commons Attribution License, which permits unrestricted use, distribution, and reproduction in any medium, provided the original work is properly cited.

\begin{abstract}
The effect of radiation on natural convection of Newtonian fluid contained in an open cavity is investigated in this study. The governing partial differential equations are solved numerically using the Alternate Direct Implicit method together with the Successive Overrelaxation method. The study is focused on studying the flow pattern and the convective and radiative heat transfer rates are studied for different values of radiation parameters, namely, the optical thickness of the fluid, scattering albedo, and the Planck number. It was found that, in the optically thin limit, an increase in the optical thickness of the fluid raises the temperature and radiation heat transfer of the fluid. However, a further increase in the optical thickness decreases the radiative heat transfer rate due to increase in the energy level of the fluid, which ultimately reduces the total heat transfer rate within the fluid.
\end{abstract}

\section{Introduction}

There are many physical phenomena in which energy exchange due to radiation plays an important role, for instance, heat transfer in furnaces and combustion chambers, solar simulators and the utilization of solar energy, flow of the earth's mantle, the flow of oxide melts during crystal growth, processing of molten glass, and the solar air receivers. Heat leakage in evacuated spaces, energy dissipation in vacuum tubes, role of air and water as coolant in power plants, and cooling of electronic and optoelectronic devices also involve the energy exchange via radiation. One of the important features of radiation heat transfer is the nature of its dependency on temperature. Review of the radiation models that exist in the literature is given by Siegel and Howell [1]. It should also be noted that the study of heat transfer in fluids which absorb and emit radiant energy becomes a complicated task due to the coupled, non-linear physical phenomena of internal radiation and natural convection. A detailed review of this coupling phenomenon of radiative and fluid transport can also be found in Modest [2].
In general, the combined mechanism of radiant and convective heat transfer in finite enclosures has received considerable attention. Larson and Viskanta [3] investigated the effects of free burning by taking a model of transient natural convection and radiation for gray diffuse walls with an arbitrary temperature in an enclosure. They showed that the difference between the temperatures of the hot and the cold walls reduces to about $1 \%$ of that of the hot wall, due to radiation effect. More interestingly, the air in the core region generally reached $33 \%$ of the hot wall temperature, whereas it obtains $13 \%$ of the hot wall temperature in the absence of radiation effect at the same time level. Lauriat [4] analyzed the effect of combined radiation and radiation phenomena for gray fluids. Bouallou and Sacadura [5] considered the porous media case of participating medium. Draoui et al. [6] further made an investigation of natural convection of participating fluids in a square enclosure. The study of flow properties for such kind of flows showed that there is a tendency of flow reduction and an increase in heat transfer for the process of combined conduction-radiation of fluids in 
enclosures. Moreover the centrosymmetric property of flow is perturbed due to the effect of radiation (see also Lauriat [4]). Salinger et al. [7] studied the effects of optical thickness and thermal gradients on the stability and structure of flows in a cylindrical container heated from below. Modest [2, page 466] indicated that there is a general misconception about lowest order differential approximation, that it may fail in optically thin limit. Rather there is some loss of accuracy only when small optically thin medium is sandwiched between hot and cold surfaces in the presence of Collimated irradiation. Derby et al. [8] examined the performance of the approximations for modeling a representative problem of heat transfer and buoyant flow in optically thick fluids. They remarked that although Rosseland diffusion had an advantage of less computational cost, an inaccuracy in the thermal boundary layer appears in it. However despite the fact that differential approximation is more costly in terms of computation due to an additional elliptic type differential equation, it gives more accurate results. Ridouane et al. [9] studied both transient and steady conditions for the effects of surface radiation on natural convection in a square enclosure heated from below and cooled from above. They showed that heat transfer across the cavity rises quickly due to active walls emissivity. Recent boundary layer study of combined mechanism of convection and radiation under different conditions can be seen in [10-14]. Liu et al. [15] used discrete ordinate method to investigate the combined mechanism of natural convection and radiation in a cavity and showed that radiative heat transfer was significantly reduced as the optical thickness was increased.

Most of the study of natural convection in enclosures has been devoted to the study of streamlines and isotherms. However, the investigation of heat function is also useful in studying the heat transfer characteristics of the phenomenon of natural convection. Kimura and Bejan [16] introduced heat function as the energy analog of stream function. Also, isotherms provide credible information about heat flow only in conduction dominated regime, whereas heatlines are locally parallel to the actual direction of energy flow in a domain. Thus heatlines study helps to comprehend the flow and temperature profile as the heat function formulation is based on identically satisfying the thermal energy equation (see also Bello-Ochende [17], Costa [18, 19], and Deng and Tang [20]). The study of heat function profile becomes more important when the natural convection flow is combined with radiation, as the inclusion of radiation raises the energy level of the fluid in domain considerably (see Larson and Viskanta [3]). A very recent study of heatlines formulation for surface radiation case in enclosures has been made by Hossain et al. [21]. Further, the problem of natural convection in open ended cavity is recently addressed by Saleem et al. [22, 23]. As far as the study of the effect of surface radiation in open cavity is concerned, the model was considered by Hinojosa et al. [24]. They investigated the Nusselt number distribution for the natural convection and surface thermal radiation in a square tilted open cavity. However, more emphasis was laid on the effect of tilt angle on the Nusselt number distributions. Also the case of conjugate natural convection with conduction and surface radiation in open cavity was studied by Nouanegue et al. [25].

The present study aims to investigate the basic flow pattern and heat transfer characteristics in open ended domain. Since, to author's knowledge, the study of participating fluids in open ended cavities is not found in literature, such kind of models may have their applications in the situations where heat is rejected via spaces between reflecting surfaces, energy transfer in vacuum tubes, and flow of air and water as coolant in power plants, where heat is mainly rejected via radiation. Thus here we consider the case of combined natural convection and radiation of Newtonian fluid in an open ended cavity, whose left wall is maintained at a higher temperature. The density of fluid with temperature is considered to vary under Boussinesq approximation. Boussinesq approximation is a better choice for laminar case of such fluids. (See also [26].) Further, it is also worth mentioning here that modeling such flows requires that the temperature of the gas does not rise enough to a level where it causes generation of large number of ions in the gas. So high energy photons due to ionic exchange do not come into account and the frequency/energy level of radiation does not vary over a wide range (Modest [2]), and gray approximation remains valid therein. Air ( $\mathrm{Pr}=$ 0.71 ) can be chosen for modeling such phenomenon (see also $[3,4,6])$ so that the ionic exchange does not take place even at high temperatures. Thus we consider a Boussinesq type fluid with gray radiant properties and consider the Rayleigh number only in the range of $2 \times 10^{5} \leq \mathrm{Ra} \leq 7 \times 10^{5}$. Attention is focused on investigating the effect of radiation parameters, namely, the optical thickness, scattering albedo, and the Planck number.

\section{Mathematical Formulation}

Consider two-dimensional flow of a viscous incompressible absorbing/emitting and scattering Boussinesq type fluid confined in an open rectangular cavity formed by the regions between two horizontal planes at $\bar{y}=0$ and $\bar{y}=Y$, and the two vertical planes at $\bar{x}=0$ and the open end along $\bar{x}=X$, where $X$ is the length and $Y$ is the height of the cavity. The left wall is assumed to be emissive, whereas its temperature is isothermally maintained at $\bar{T}_{H}$. The temperature of the fluid that enters the cavity region is supposed to be at $\bar{T}_{C}$ at $\bar{t}=0$ (where $\bar{T}_{H}>\bar{T}_{C}$ ). In order to represent the temperature at the open end, we use the subscripts "in" for incoming and "out" for outgoing fluid, respectively. Flow configuration and dynamical boundary conditions are shown in Figure 1.

Here, $\bar{u}$ and $\bar{v}$ are the components of velocity along the $\bar{x}$ and $\bar{y}$ axes, respectively, $\bar{T}$ is the fluid temperature, and $g$ is the magnitude of acceleration due to gravity whereas $\epsilon_{i}$, $(i=i, 2,3)$ is the emissivity of left, top, and bottom walls, respectively. The unsteady motion of incompressible fluid and the equations for conservation of mass, momentum, and 


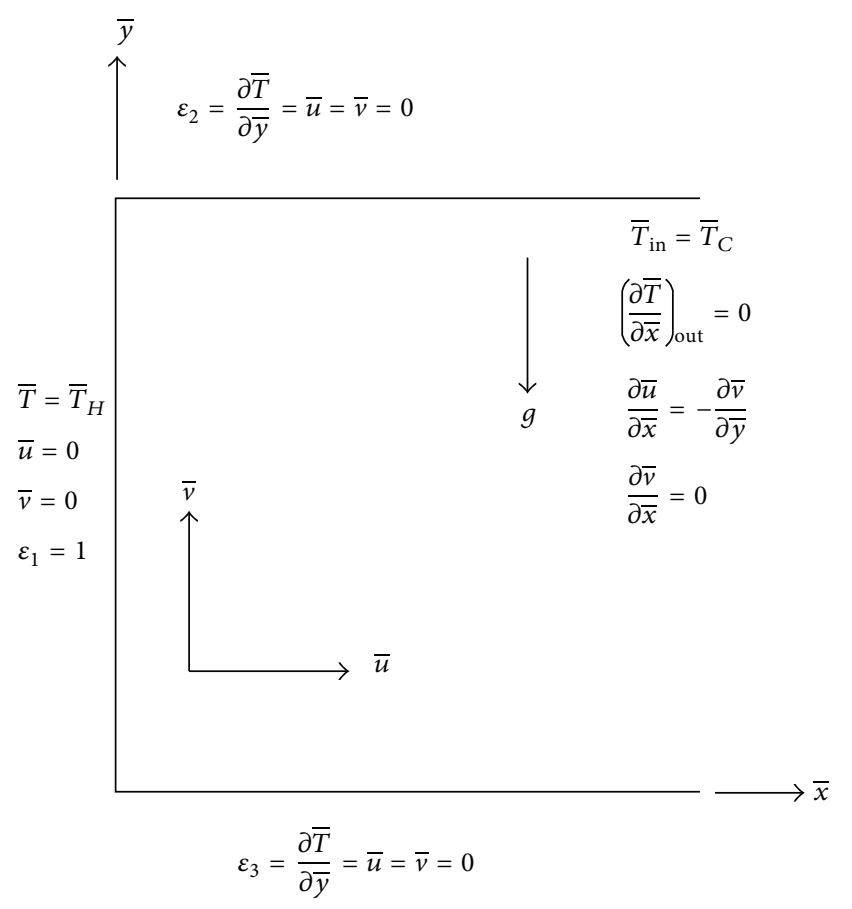

FIgURE 1: Flow configuration in coordinate system.

energy in two-dimensional Cartesian coordinate system are given by

$$
\begin{gathered}
\frac{\partial \bar{u}}{\partial \bar{x}}+\frac{\partial \bar{v}}{\partial \bar{y}}=0, \\
\frac{\partial \bar{u}}{\partial \bar{t}}+\bar{u} \frac{\partial \bar{u}}{\partial \bar{x}}+\bar{v} \frac{\partial \bar{u}}{\partial \bar{y}}=-\frac{1}{\rho} \frac{\partial \bar{p}}{\partial \bar{x}}+\nu\left(\frac{\partial^{2} \bar{u}}{\partial \bar{x}^{2}}+\frac{\partial^{2} \bar{u}}{\partial \bar{y}^{2}}\right), \\
\frac{\partial \bar{v}}{\partial \bar{t}}+\bar{u} \frac{\partial \bar{v}}{\partial \bar{x}}+\bar{v} \frac{\partial \bar{v}}{\partial \bar{y}}=-\frac{1}{\rho} \frac{\partial \bar{p}}{\partial \bar{y}}+\nu\left(\frac{\partial^{2} \bar{v}}{\partial \bar{x}^{2}}+\frac{\partial^{2} \bar{v}}{\partial \bar{y}^{2}}\right)+g \beta\left(\bar{T}-\bar{T}_{C}\right), \\
\rho C_{p}\left(\frac{\partial \bar{T}}{\partial \bar{t}}+\bar{u} \frac{\partial \bar{T}}{\partial \bar{x}}+\bar{v} \frac{\partial \bar{T}}{\partial \bar{y}}\right)=k\left(\frac{\partial^{2} \bar{T}}{\partial \bar{x}^{2}}+\frac{\partial^{2} \bar{T}}{\partial \bar{y}^{2}}\right)-\nabla \cdot \bar{q}_{r},
\end{gathered}
$$

where $v$ is the kinematic viscosity, $\beta$ is the coefficient of volume expansion, $\rho$ is the density, $C_{p}$ is the molar specific heat at constant pressure, $k$ is the coefficient of thermal conductivity, $\bar{t}$ is the time, and $\bar{q}_{r}$ is the radiation flux vector in two dimensions. We have considered semitransparent fluid with gray radiant properties. The equation of radiant energy for optically thick absorbing/emitting and scattering fluid is thus given by (see [4-6])

$$
\frac{\partial^{2} \bar{G}}{\partial \bar{x}^{2}}+\frac{\partial^{2} \bar{G}}{\partial \bar{y}^{2}}=3 \alpha_{m}^{2}\left(1-\Omega_{0}\right)\left\{\bar{G}-\sigma\left(\bar{T}-\bar{T}_{C}\right)^{4}\right\}
$$

where $\alpha_{m}$ is mean extinction coefficient, $\Omega_{0}$ is scattering albedo, and $\sigma$ is Stefan-Boltzmann constant. The boundary conditions are expressed as follows:

$$
\begin{aligned}
& \bar{t}<0 \\
& \bar{u}=\bar{v}=\bar{T}=\bar{G}=0, \quad 0 \leq \bar{y} \leq Y, \quad 0 \leq \bar{x} \leq X,
\end{aligned}
$$

$\bar{t} \geq 0$. First we describe the boundary conditions at the solid walls. The velocity components at the solid walls are given by no slip condition

$$
\bar{u}=\bar{v}=0 .
$$

The temperature of the left wall is given by

$$
\bar{T}=\bar{T}_{H}, \quad \bar{x}=0, \quad 0 \leq \bar{y} \leq Y,
$$

whereas the temperature at the nonisothermal walls is given by

$$
k \frac{\partial \bar{T}}{\partial \bar{y}}+\frac{1}{3 \alpha_{m}} \frac{\partial \bar{G}}{\partial \bar{y}}=0, \quad \bar{y}=0, Y, \quad 0 \leq \bar{x} \leq X .
$$

Finally the boundary conditions at the solid walls for thermal radiation are

$$
\frac{\partial \bar{G}}{\partial \bar{n}_{i}}=\frac{3 \alpha_{m} \epsilon_{i}}{4-2 \epsilon_{i}}\left\{\bar{G}-4 \sigma\left(\bar{T}_{i}-\bar{T}_{C}\right)^{4}\right\} .
$$

Here, $\bar{n}$ is the direction normal to the wall and $i=1,2,3$, respectively, represent the left, top, and bottom wall as labeled in Figure 1. (See [6].)

To set up the open end boundary conditions, notice that the velocity component $\bar{u}, \bar{v}$ should satisfy the continuity equation at the open end. Moreover, $\partial \bar{v} / \partial \bar{x}=0$ is a physically more realistic condition for vertical velocity at the open end as compared to $\bar{v}=0$, as the fluid may not necessarily leave the domain horizontally. Further, $(\partial \bar{T} / \partial \bar{x})_{\text {out }}=0$ is found to be a suitable condition at the open end for temperature. Most recently, these conditions are also addressed by the same authors (Saleem et al. [22, 23] and see the references therein). Further, since it is required to confine the effects of wall radiation within the cavity, this suggests that the divergence of radiation intensity at the open end for thermal radiation should be zero. Thus the boundary conditions at the open end of the cavity are summarized by the following equations

$$
\begin{gathered}
\frac{\partial \bar{u}}{\partial \bar{x}}=-\frac{\partial \bar{v}}{\partial \bar{y}}, \quad \frac{\partial \bar{v}}{\partial \bar{x}}=\frac{\partial \bar{G}}{\partial \bar{x}}=0, \\
\left(\frac{\partial \bar{T}}{\partial \bar{x}}\right)_{\text {out }}=0, \quad \bar{T}_{\text {in }}=\bar{T}_{C}, \quad \bar{x}=X, \quad 0 \leq \bar{y} \leq Y .
\end{gathered}
$$

In order to transform the problem into stream vorticity form, we define the stream function and vorticity by the following relations:

$$
\begin{gathered}
\bar{\omega}=\frac{\partial \bar{v}}{\partial \bar{x}}-\frac{\partial \bar{u}}{\partial \bar{y}}, \\
\bar{u}=\frac{\partial \bar{\psi}}{\partial \bar{y}}, \quad \bar{v}=-\frac{\partial \bar{\psi}}{\partial \bar{x}} .
\end{gathered}
$$


Further introduce the nondimensional variables by using the following transformations:

$$
\begin{gathered}
x=\frac{\bar{x}}{Y}, \quad y=\frac{\bar{y}}{Y}, \quad t=\frac{\bar{t} \alpha}{Y^{2}}, \quad u=\frac{\overline{u Y}}{\alpha}, \\
v=\frac{\bar{v} Y}{\alpha}, \quad \psi=\frac{\bar{\psi}}{\alpha}, \quad \omega=\frac{\bar{\omega} Y^{2}}{\alpha}, \quad \Delta \bar{T}=\bar{T}_{H}-\bar{T}_{C}, \\
\bar{T}_{0}=\frac{\bar{T}_{H}+\bar{T}_{C}}{2}, \quad \theta=\frac{\bar{T}-\bar{T}_{C}}{\Delta \bar{T}}, \quad A=\frac{Y}{X}, \\
\theta_{0}=\frac{\bar{T}_{0}}{\Delta \bar{T}}, \quad q_{r}=\frac{\bar{q}_{r} A}{\sigma \bar{T}_{0}^{4}}, \quad G=\frac{\bar{G}}{\sigma \bar{T}_{0}^{4}}, \quad \tau_{0}=\alpha_{m} X,
\end{gathered}
$$

where $x, y$ are the nondimensional coordinate axis, $u, v$ are the nondimensional velocity components, $\psi$ and $\omega$ are the nondimensional stream and vorticity functions, $t$ is the time, $\bar{T}_{0}$ is the reference temperature, $\theta$ is the nondimensional temperature, $\theta_{0}$ is the mean temperature in nondimensional form, $G$ is the dimensionless radiant energy, and $\tau_{0}$ is the optical thickness. The treatment of radiative flux divergence in (2) is based on two-dimensional differential approximation. The nondimensional form of radiative flux and its divergence are given by the following relations (see also $[1,2,4-6]$ ):

$$
q_{r, x}=-\frac{1}{3 \tau_{0}} \frac{\partial G}{\partial x}, \quad q_{r, y}=-\frac{1}{3 \tau_{0}} \frac{\partial G}{\partial y},
$$

where $q_{r, x}$ and $q_{r, y}$ are the radiative fluxes along the $x$ and $y$ components, respectively. Thus the governing equations in nondimensional form finally become

$$
\begin{gathered}
\frac{\partial^{2} \psi}{\partial x^{2}}+\frac{\partial^{2} \psi}{\partial y^{2}}=-\omega, \\
\frac{\partial \omega}{\partial t}+u \frac{\partial \omega}{\partial x}+v \frac{\partial \omega}{\partial y}=\operatorname{Pr}\left(\frac{\partial^{2} \omega}{\partial x^{2}}+\frac{\partial^{2} \omega}{\partial y^{2}}\right)+\operatorname{RaPr} \frac{\partial \theta}{\partial x} \\
\frac{\partial \theta}{\partial t}+u \frac{\partial \theta}{\partial x}+v \frac{\partial \theta}{\partial y}=\left(\frac{\partial^{2} \theta}{\partial x^{2}}+\frac{\partial^{2} \theta}{\partial y^{2}}\right)+\frac{1}{3 N_{\mathrm{CR}}}\left(\frac{\partial^{2} G}{\partial x^{2}}+\frac{\partial^{2} G}{\partial y^{2}}\right), \\
\frac{\partial^{2} G}{\partial x^{2}}+\frac{\partial^{2} G}{\partial y^{2}}=3 \tau_{0}^{2} A^{2}\left(1-\Omega_{0}\right)\left(G-4 \frac{\theta^{4}}{\theta_{0}^{4}}\right)
\end{gathered}
$$

with the following boundary conditions:

$$
\begin{aligned}
& t<0 \\
& u=v=\psi=\omega=\theta=G=0, \quad 0 \leq y \leq 1, \quad 0 \leq x \leq \frac{1}{A} \\
& t \geq 0 \\
& u=v=\psi=0, \quad \omega=-\frac{\partial u}{\partial y}, \quad y=0, \quad 0 \leq x \leq \frac{1}{A}, \\
& u=v=\psi=0, \quad \omega=-\frac{\partial u}{\partial y}, \quad y=1, \quad 0 \leq x \leq \frac{1}{A},
\end{aligned}
$$

$$
\begin{aligned}
& u=v=\psi=0, \quad \omega=\frac{\partial v}{\partial x}, \quad \theta=1, \quad x=0, \quad 0 \leq y \leq 1, \\
& \frac{\partial u}{\partial x}=-\frac{\partial v}{\partial y}, \quad \omega_{\text {in }}=\theta_{\text {in }}=G_{\text {in }}=0, \quad x=\frac{1}{A}, \quad 0 \leq y \leq 1, \\
& \frac{\partial v}{\partial x}=\left(\frac{\partial \omega}{\partial x}\right)_{\text {out }}=\left(\frac{\partial \theta}{\partial x}\right)_{\text {out }}=\left(\frac{\partial G}{\partial x}\right)_{\text {out }}=0, \\
& x=\frac{1}{A}, \quad 0 \leq y \leq 1,
\end{aligned}
$$

where

$$
\operatorname{Pr}=\frac{\nu}{\alpha}, \quad \operatorname{Ra}=\frac{g \beta \Delta \bar{T} H^{3}}{\alpha \nu}, \quad N_{\mathrm{CR}}=\frac{k \alpha_{m} \Delta \bar{T}}{\sigma \bar{T}_{0}^{4}}
$$

are, respectively, the Prandtl number, the Rayleigh number, and the Planck number (also known as conduction-radiation parameter). The temperature boundary conditions for horizontal walls and radiation energy conditions at the three solid walls in nondimensional form are now given by $([1,2,4-6])$

$$
\begin{aligned}
\frac{\partial \theta}{\partial y}+\frac{1}{3 N_{\mathrm{CR}}} \frac{\partial G}{\partial y} & =0, \quad y=0,1, \quad 0 \leq x \leq \frac{1}{A}, \\
\frac{\partial G}{\partial n_{i}} & =\frac{3 \tau_{0} A \epsilon_{i}}{4-2 \epsilon_{i}}\left(G-4 \frac{\theta_{i}^{4}}{\theta_{0}^{4}}\right) .
\end{aligned}
$$

Heatlines are a measure of the path followed by the heat function across the flow region. Following the formulation of heat function defined by Kimura and Bejan [16], we construct a Poisson-type heat function equation that identically satisfies the energy transport equation (2) given by (see also [15-19])

$$
\begin{gathered}
\frac{\partial \bar{H}}{\partial \bar{y}}=\rho C_{p} \bar{u}\left(\bar{T}-\bar{T}_{C}\right)-k \frac{\partial \bar{T}}{\partial \bar{x}}+\bar{q}_{r, x}, \\
-\frac{\partial \bar{H}}{\partial \bar{x}}=\rho C_{p} \bar{v}\left(\bar{T}-\bar{T}_{C}\right)-k \frac{\partial \bar{T}}{\partial \bar{y}}+\bar{q}_{r, y} .
\end{gathered}
$$

Now defining $H=\bar{H} / k \Delta \bar{T}$ as the nondimensional heat function and making use of (12), we get the following definition of heat function:

$$
\frac{\partial^{2} H}{\partial x^{2}}+\frac{\partial^{2} H}{\partial y^{2}}=u \frac{\partial \theta}{\partial y}+v \frac{\partial \theta}{\partial x}-\theta \omega .
$$

The wall boundary conditions for the heatlines are followed by the definition of heat function given by (17) (see also [1519]):

$$
\begin{array}{ccc}
\frac{\partial H}{\partial y}=-\frac{\partial T}{\partial x}, & y=0,1, & 0 \leq x \leq \frac{1}{A}, \\
\frac{\partial H}{\partial x}=\frac{\partial T}{\partial y}, & x=0, & 0 \leq y \leq 1, \\
\frac{\partial H}{\partial x}=0, & x=\frac{1}{A}, & 0 \leq y \leq 1 .
\end{array}
$$


Finally we define the heat transfer from the left wall. When energy transfer takes place in combined mode, the net heat transfer rate is also defined as the combined mechanism of these modes (Lauriat [4]). Thus for combined mechanism of convection and radiation the net heat transfer for solid wall boundaries can be defined by the following relations (see [4$6,21])$ :

$$
\begin{gathered}
\mathrm{Nu}_{C}=-\left(\frac{\partial \theta}{\partial x}\right)_{x=0}, \quad \mathrm{Nu}_{R}=-\frac{1}{3 N_{\mathrm{CR}}}\left(\frac{\partial G}{\partial x}\right)_{x=0}, \\
\mathrm{Nu}=\mathrm{Nu}_{C}+\mathrm{Nu}_{R} .
\end{gathered}
$$

From here it follows that the average Nusselt number is defined as

$$
\begin{aligned}
\overline{\mathrm{Nu}} & =\overline{\mathrm{Nu}}_{\mathrm{C}}+\overline{\mathrm{Nu}}_{R}=\int_{0}^{1} \mathrm{Nu} d y \\
& =\int_{0}^{1}\left[\left(-\frac{\partial \theta}{\partial x}\right)_{x=0}+\left(-\frac{1}{3 N_{\mathrm{CR}}} \frac{\partial G}{\partial x}\right)_{x=0}\right] d y,
\end{aligned}
$$

where $d y$ is the element of length $Y$ along the wall. Equations (13) to (16) are solved with the boundary conditions given by (17) and (19) to solve the system. The heat lines are given by (21) whereas the average heat transfer is calculated from (24).

\section{Method of Solution}

The flow is developed by coupling of buoyancy term in (14), which is updated by (15) and (16), stream function is obtained by coupling of (13) and (14), and finally velocity profile is updated using the nondimensional form of (10). The solution is complemented with the implementation of boundary conditions given in (17) and (19). The stream function equation (13) is solved using Successive Over Relaxation (SOR) method with residual tolerance of order $10^{-5}$. With $Y$ as the reference height of the cavity, we have considered a uniform grid of size $h=Y /(J-1)$, where $J$ is the maximum number of grids along coordinate axes. Throughout the computation we take $Y=1$. The time dependent vorticity transport equation (14) and thermal conduction-convection equation (15) are solved using the Alternate Direct Implicit method. The details of the proposed method are also given in [21-23, 27]. A simple discretization procedure given by Bello-Ochende [17] is adopted for the solution of radiation equation (16) and heat function equation given by (21). As a simple case, all computations are performed for square cavity. That is, $A=1$. Now in order to meet the criteria of convergence to reach the steady state, we define the error bound in the computed value of a variable $F$, by the relation

$$
\left|\frac{F_{(i, j)}^{m+1}-F_{(i, j)}^{m}}{F_{(i, j)}^{m}}\right|<10^{-8},
$$

where the superscript $m$ refers to the number of time steps and $(i, j)$ is a grid location on coordinate axes. A grid dependence study has been carried out for the choice of suitable number of grid points whose results are shown in Figure 2.

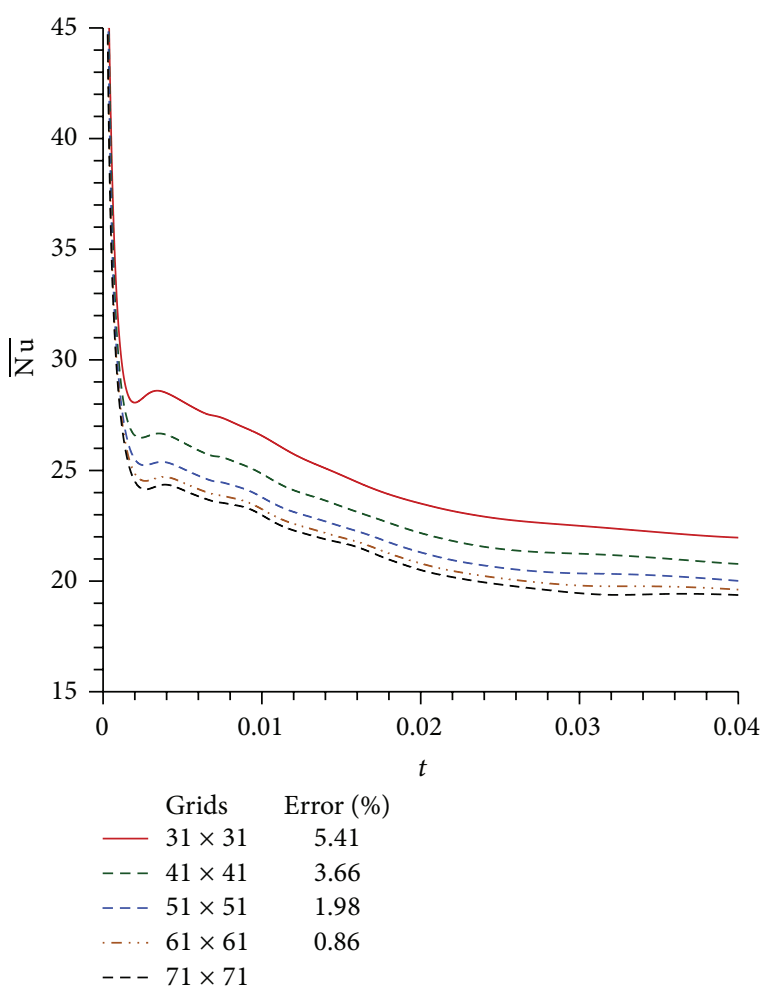

FIGURE 2: Average heat transfer rate of the heated wall against time for various grid choices at $\mathrm{Ra}=5 \times 10^{5}, \mathrm{Pr}=0.7$, and $\tau_{0}=\Omega_{0}=$ $N_{\text {CR }}=0.5$.

Figure 2 shows the result of grid dependence study at $\mathrm{Ra}=$ $5 \times 10^{5}, \tau_{0}=\Omega_{0}=N_{\mathrm{CR}}=0.5$ for the choice of appropriate mesh size $h$. For any variable $F$, we define the relative error $\%$ between the computed values taking different grid points as given by

$$
\text { Error\% }=\left|\frac{F_{(\kappa+10, \kappa+10)}-F_{\kappa, \kappa}}{F_{\kappa, \kappa}}\right| \text {, }
$$

where $F_{\kappa, \kappa}$ is previously calculated value of a variable for $(\kappa \times \kappa)$ grid points. As a demonstration, Figure 2 shows the percentage error in the values obtained from the difference between the computed values of the Average Nusselt number, for different choices of mesh points. It can be seen that the maximum error between mesh sizes $61 \times 61$ and $71 \times 71$, in terms of $\overline{\mathrm{Nu}}$, drops to less than $1 \%$. Thus a mesh of $61 \times$ 61 is considered sufficient for the entire computation. The reduction in relative error justifies the grid independence of the solution. In order to further check the validity for the solution method of the proposed model, we have also revisited the work of Draoui et al. [6] using the solid wall conditions and the temperature and radiation condition given by [6] for the right wall. The comparison of streamlines, isotherms, and heatlines, for $\mathrm{Ra}=10^{5}, \mathrm{Pr}=0.7, \tau_{0}=1, \Omega_{0}=$ 0.2 , and $N_{\mathrm{CR}}=0.1$, is shown in Figure 3. From this figure we assert that the numerical solution we obtained agrees 


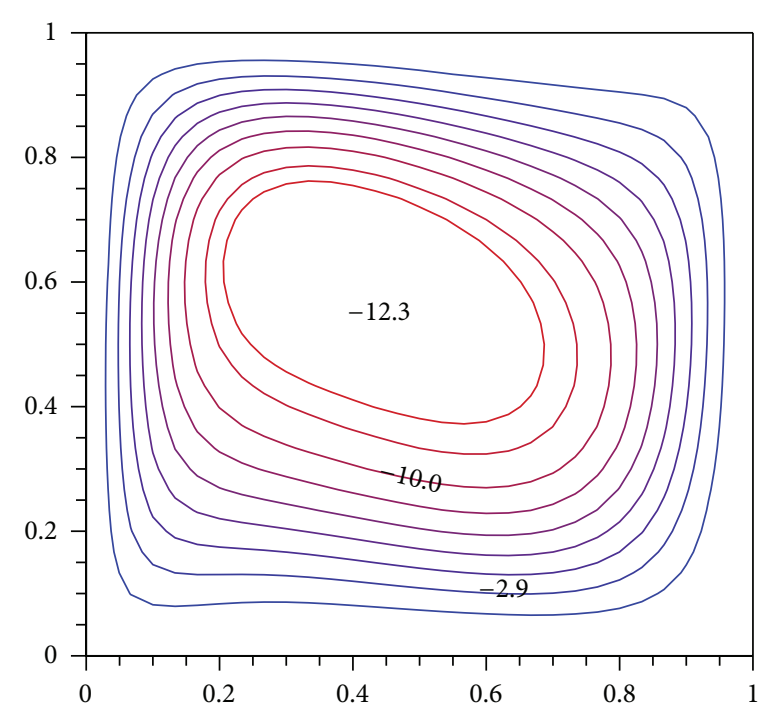

(a)

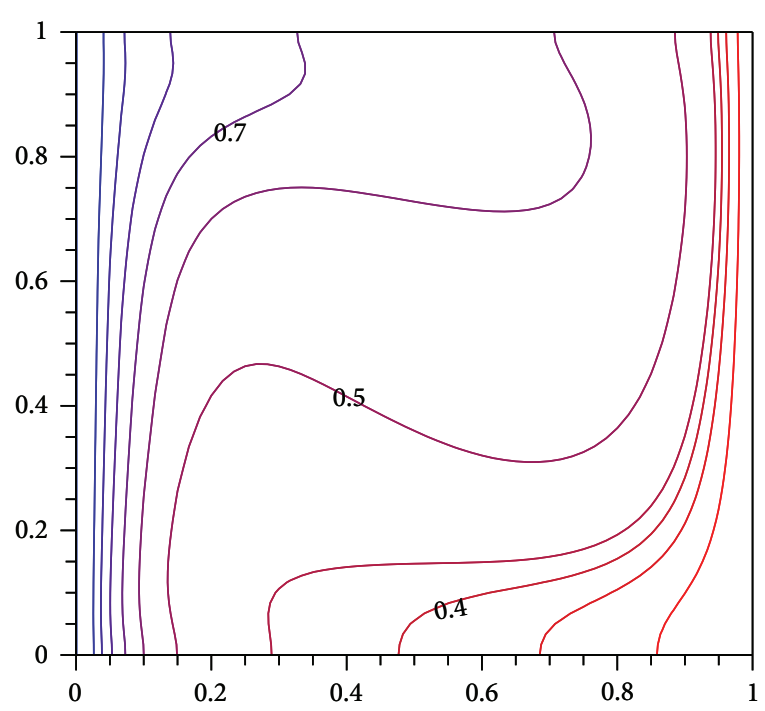

(b)

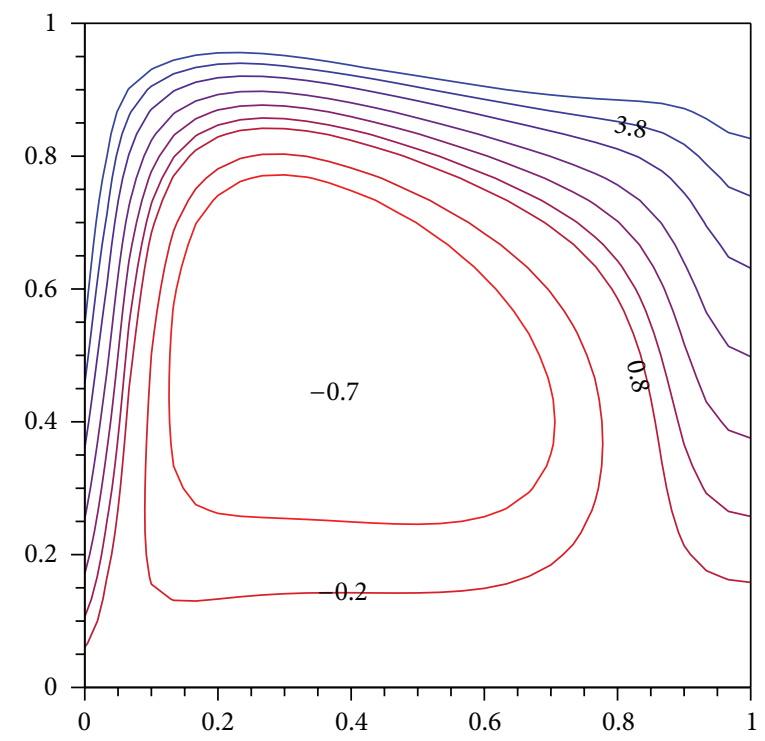

(c)

Figure 3: Comparison of (a) streamlines and (b) isotherms. (c) Heat lines with the results of Draoui et al. [6] for $\mathrm{Ra}=10^{5}, \mathrm{Pr}=0.7, \tau_{0}=1$, $\Omega_{0}=0.2$, and $N_{\mathrm{CR}}=0.1$ with the conditions given by [6].

well with the existing literature of heatlines. Intel $1.83 \mathrm{GHz}$ processing machine is used for the entire computation.

\section{Results and Discussion}

We have considered combined natural convection and radiation phenomena for participating fluid confined in an open square cavity. The left wall is considered at a temperature higher than that of the fluid entering from the ambient region. The effect of relevant radiation parameters, namely, the optical thickness, scattering albedo, and Planck number, on the flow profile and heat transfer rate has been numerically studied. The result is presented graphically in terms of streamlines, isotherms, heatlines, and heat transfer rate for different values of these governing physical parameters.

4.1. Effect of Optical Thickness. Figure 4 represents the selected results of steady state pattern of streamlines while $\mathrm{Ra}=7 \times 10^{5}, \Omega_{0}=0.2$, and $N_{\mathrm{CR}}=0.1$ for $(\mathrm{a}) \tau_{0}=0.0$, (b) $\tau_{0}=$ 0.5 , and (c) $\tau_{0}=1.0$, respectively. The result of Figure 4(a) shows the streamlines in the absence of radiation effects. However, with the increase in optical thickness, the flow in the core region decreases as shown in Figure 4(b); rather it is minimum at this value of $\tau_{0}$. By further increasing the optical thickness, the flow develops into a multicell pattern due to the dominance of radiation effects into the core region. It may be due to the reason that the fluid in the core region experiences 


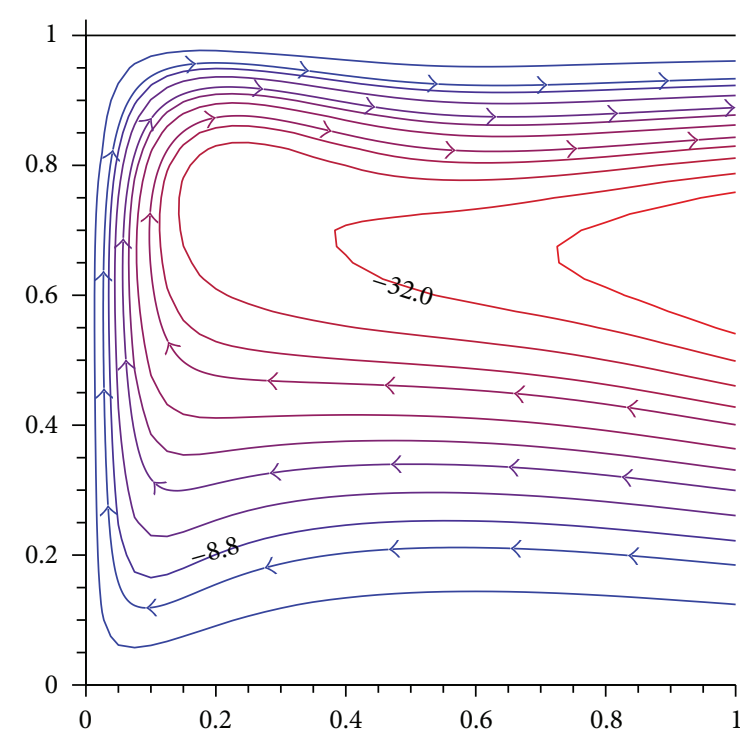

(a)

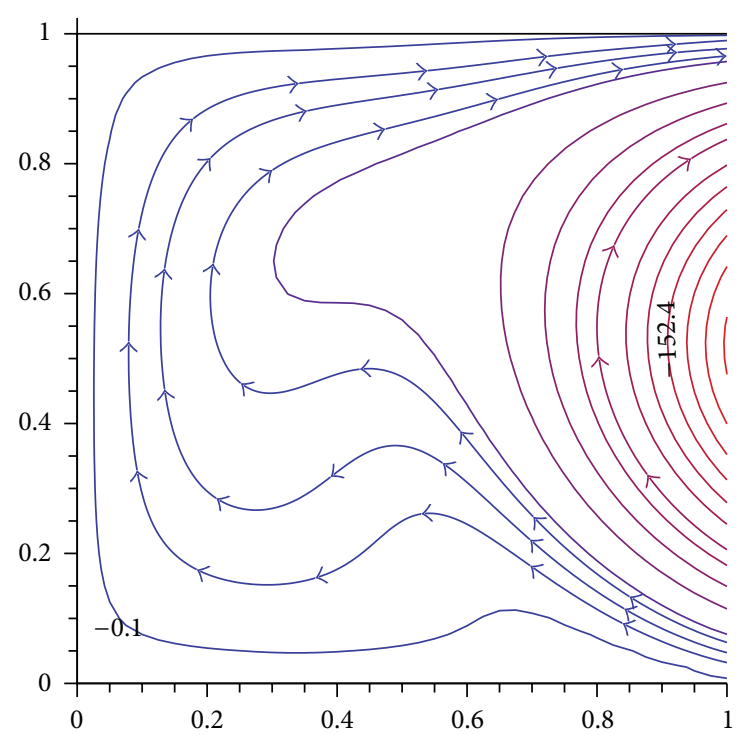

(b)

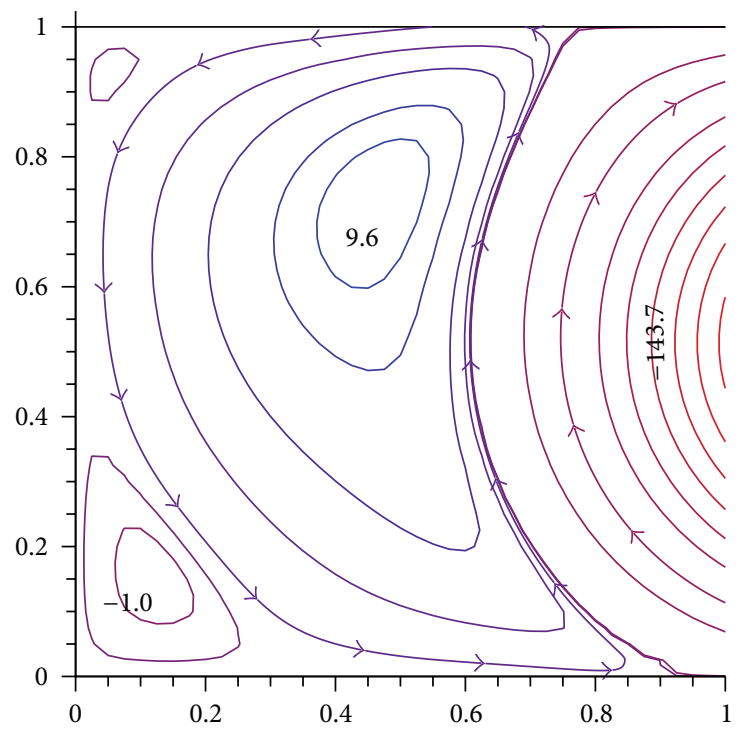

(c)

FIGURE 4: Steady state pattern of streamlines for $\mathrm{Ra}=7 \times 10^{5}, \Omega_{0}=0.2$, and $N_{\mathrm{CR}}=0.1$ at (a) $\tau_{0}=0.0$, (b) $\tau_{0}=0.5$, and (c) $\tau_{0}=1.0$.

a force from the incoming fluid, whereas the recirculation is higher in the half open vortex close to the opening. This counteracting mechanism develops a multicellular pattern as shown in Figure 4(c). Figure 5 shows the isotherms at these values of optical thickness. Figure 5(a) shows that, at $\tau_{0}=$ 0.0 , the isotherms are clustered close to the heated wall. It is due to the dominance of convection in the boundary layer region. However with the increase in optical thickness, the boundary layer thickness increases. This effect is qualitatively in agreement with the case of completely confined enclosures. The radiation energy adds to the total energy of isotherms and thus the high energy isotherms shift towards the core region as shown in Figure 5(b). At $\tau_{0}=1.0$, the whole flow domain has the isotherms nearly equal to unity due to the dominance of radiant energy, shown in Figure 5(c). This might well be understood also from the heatlines pattern of Figure 6.
Figure 6 shows the heatlines while $\mathrm{Ra}=7 \times 10^{5}, \Omega_{0}=$ 0.2 , and $N_{\mathrm{CR}}=0.1$ for (a) $\tau_{0}=0.0$, (b) $\tau_{0}=0.5$, and (c) $\tau_{0}=1.0$, respectively. Obviously, at $\tau_{0}=0.0$, there is no contribution of radiation in heat transfer and fluid flow. All fluid entering the flow domain absorbs heat from the solid wall and heatlines are negative throughout the flow region with higher values close to the solid wall as shown in Figure 6(a). The flow region, in which the recirculation is weaker (as in Figure 4(b)), is the region which absorbs heat at $\tau_{0}=0.5$, given in Figure $6(\mathrm{~b})$. The positive part of the heatlines pattern is the region of fluid with higher energy level. Figure 6(c) shows the heatlines pattern at $\tau_{0}=$ 1.0. This clearly indicates the high energy level of fluid due to dominance of radiation effect. The heatlines are positive throughout the region, which indicates that the fluid in the region is at high energy level and emitting heat. The effect of 


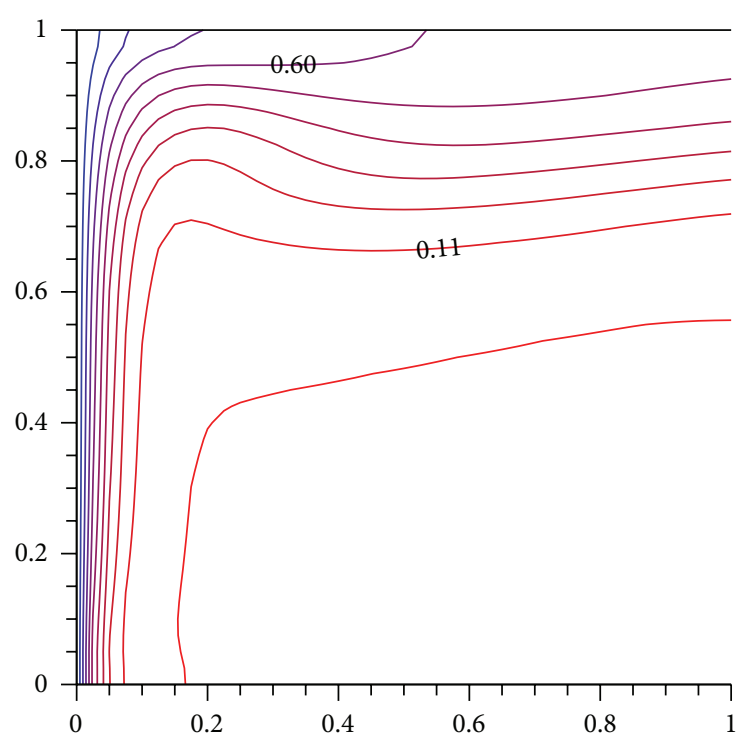

(a)

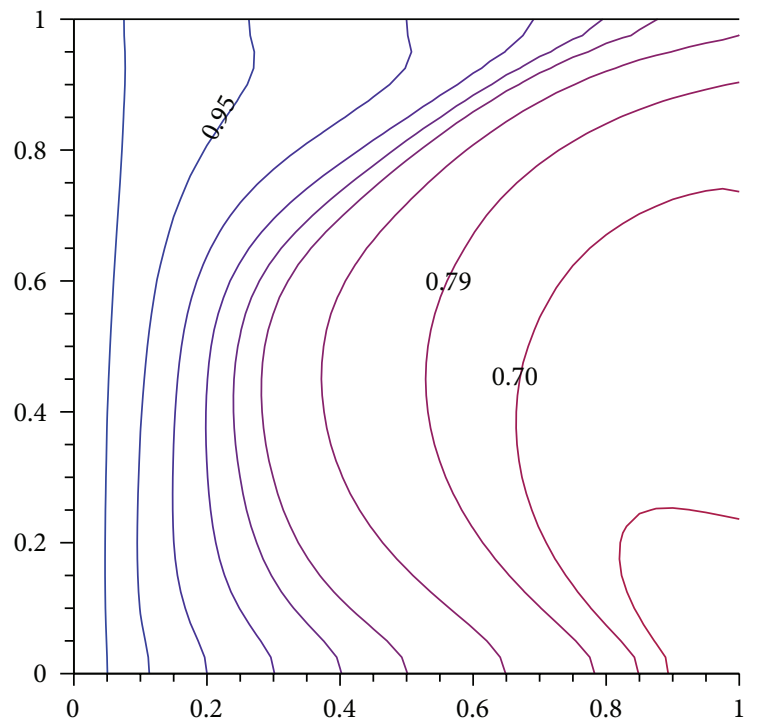

(b)

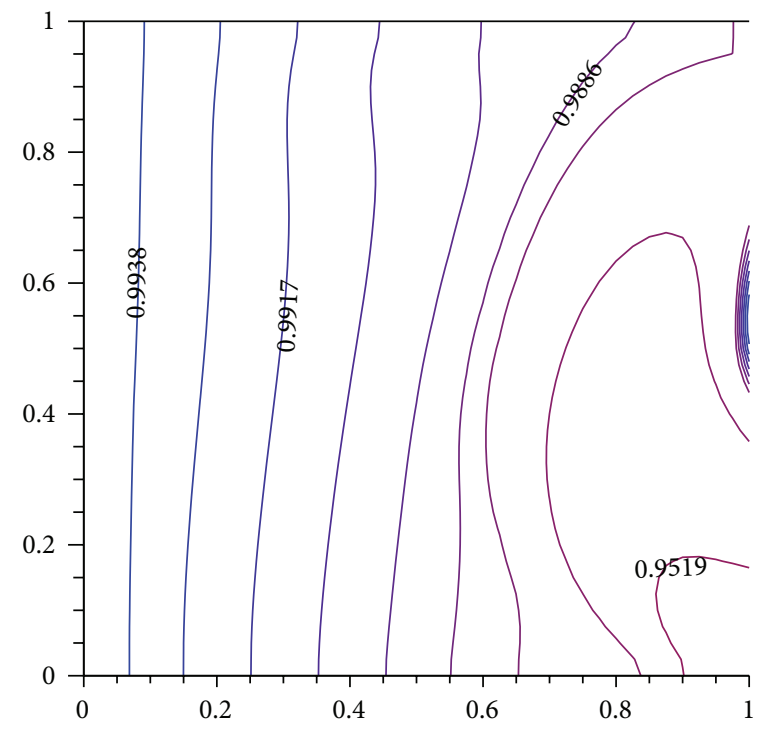

(c)

FIGURE 5: Isotherms for $\mathrm{Ra}=7 \times 10^{5}, \Omega_{0}=0.2$, and $N_{\mathrm{CR}}=0.1$ at (a) $\tau_{0}=0.0$, (b) $\tau_{0}=0.5$, and (c) $\tau_{0}=1.0$.

optical thickness on heat transfer is described in Figure 7. It can be discerned that the overall heat transfer $\mathrm{Nu}$ pattern is dominated by the radiation heat transfer $\mathrm{Nu}_{R}$. In particular, overall heat transfer is almost the same as radiative heat transfer beyond $\tau_{0}>0.5$. Heat transfer is maximum at $\tau_{0}=0.5$, when the flow in the core region is very weak. The contribution of convective heat transfer asymptotically vanishes with the increase in the contribution of optical thickness. At $\tau_{0}=1.25$, both the radiative and convective heat transfer rates coincide with "0," beyond which the negative heat transfer signifies the energy level of fluid due to the fact that contribution of radiant energy is so high that it serves to reject heat to the solid wall. However, the smooth curve, for $\tau_{0}>1.75$, shows that the flow pattern and transfer rates are not much affected beyond $\tau_{0}=1.75$.
4.2. Effect of Scattering Albedo. Scattering albedo is a measure of radiative participation of the fluid. $0 \leq \Omega_{0} \leq 1$ shifts the fluid regime from perfectly participating to nonparticipating. Figure 8 shows the average total heat transfer against time at $\mathrm{Ra}=2 \times 10^{5}, \tau_{0}=0.5$, and $N_{\mathrm{CR}}=0.2$ for different values of scattering albedo $\Omega_{0}$. The right hand side of (16) signifies that the fluid is perfectly transmitting at $\Omega_{0}=0$. This justifies that with the increase in the value of $\Omega_{0}$, the scattering increases, which enhances the effect of convective heat transfer; ultimately $\mathrm{Nu}_{C}$ increases whereas the radiative heat transfer decreases and the decrease seems more prominent because $\Omega_{0}=1$ decouples the radiation equation from the thermal energy. Thus, at $\Omega_{0}=1$, the radiative heat transfer is zero. Figure 8 signifies that $\Omega_{0}$ has no significant effect on the overall heat transfer $0 \leq \Omega_{0} \leq 0.75$; 


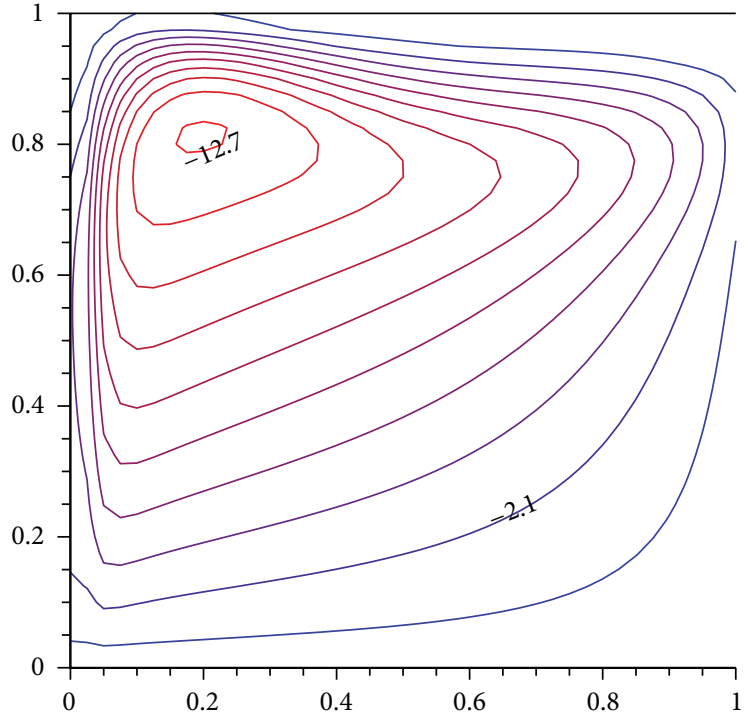

(a)

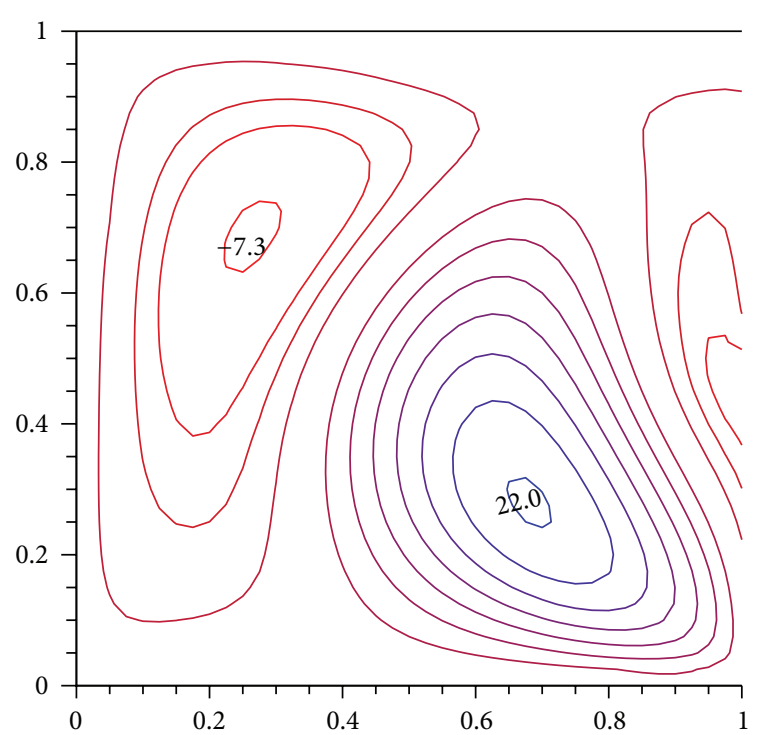

(b)

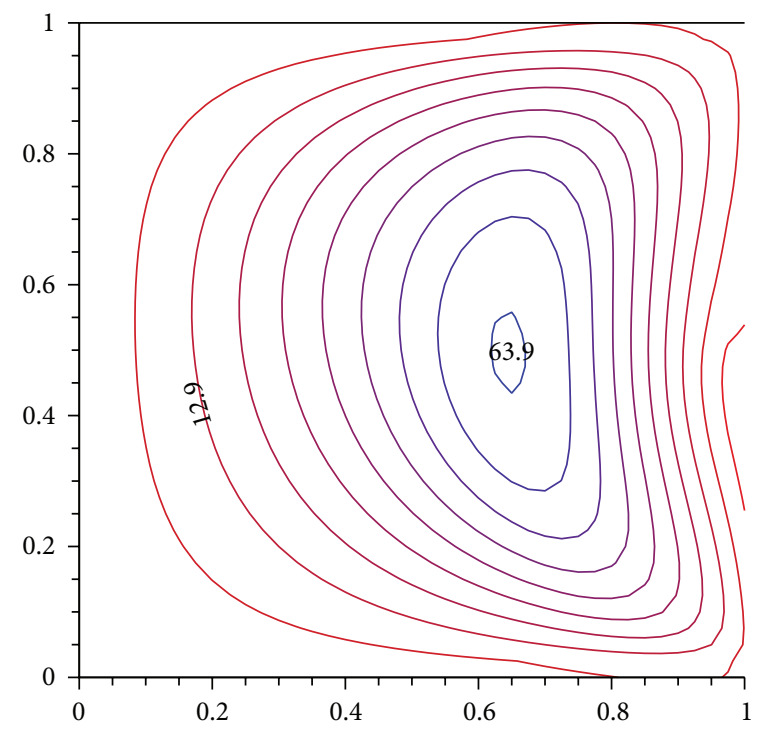

(c)

FIgURE 6: Heat lines for $\mathrm{Ra}=7 \times 10^{5}, \Omega_{0}=0.2$, and $N_{\mathrm{CR}}=0.1$ at (a) $\tau_{0}=0.0$, (b) $\tau_{0}=0.5$, and (c) $\tau_{0}=1.0$.

rather, there is a considerable decrease only between $\Omega_{0}=$ 0.75 and $\Omega_{0}=1$ due to the reason that at $\Omega_{0}=1$ the fluid surface is perfectly scattering, and the contribution of $\mathrm{Nu}_{R}$ vanishes at this value of $\Omega_{0}$.

4.3. Effect of Planck Number. Planck number represents the ratio of conduction to radiation effects on the fluid. The greater the value of $N_{\mathrm{CR}}$ is, the lesser the effect of radiation on the fluid will be. Figure 9 shows the streamlines at $\mathrm{Ra}=5 \times$ $10^{5}, \Omega_{0}=0.2$, and $\tau_{0}=1.0$ for (a) $N_{\mathrm{CR}}=0.2$, (b) $N_{\mathrm{CR}}=2.0$, and (c) $N_{\mathrm{CR}}=4.0$, respectively. At $N_{\mathrm{CR}}=0.2$, the radiation effect is dominant and the flow in the core region is weaker. Rather, a multicellular pattern appears due to the reason that the lower energy fluid is trapped by the recirculating fluid close to the opening. However, at $N_{\mathrm{CR}}=2.0$, the two effects are comparable, and the flow in the core region develops into a unicellular pattern with maximum value of the flow at the opening. At $N_{\mathrm{CR}}=4.0$, the conduction effect is dominant and the flow further decreases. The flow in Figure 9(c) is weaker than the one noticed in Figure 9(b), because of the gradual exclusion of radiant energy from the flow. Figure 10 shows the heatlines at $\mathrm{Ra}=5 \times 10^{5}, \Omega_{0}=0.2$, and $\tau_{0}=1.0$ for (a) $N_{\mathrm{CR}}=0.2$, (b) $N_{\mathrm{CR}}=2.0$, and (c) $N_{\mathrm{CR}}=4.0$. Obviously at $N_{\mathrm{CR}}=0.2$, the radiant energy is a dominant factor in the flow, because the fluid in most of the domain is at higher energy level, shown by positive values of heatlines. However, the heatline labeled -6 at the opening signifies that there is a small portion of the incoming fluid that absorbs heat from the flow domain. Figures 10 (b) and 10 (c) show that as the value of Planck number increases, more and more fluid in the 


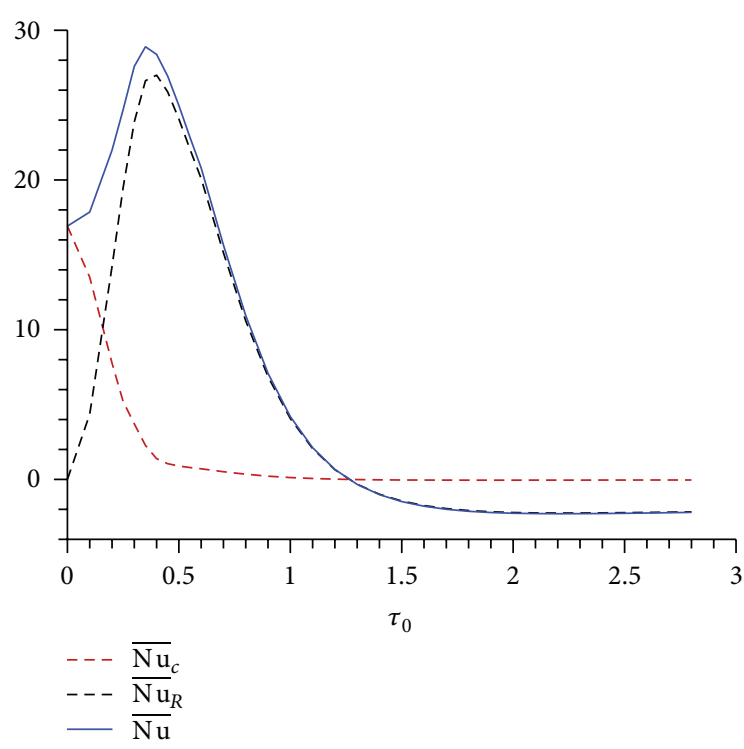

FIGURE 7: Average heat transfer rates against various values of optical thickness $\tau_{0}$ at $\mathrm{Ra}=7 \times 10^{5}, \Omega_{0}=0.2$, and $N_{\mathrm{CR}}=0.1$.

core region absorbs heat by conduction due to decrease in the energy level of the fluid in the core region.

Finally Figure 11 shows the convective, radiative, and total heat transfer as a function of $N_{\mathrm{CR}}$ while $\tau_{0}=1, \Omega_{0}=0.2$, and $\mathrm{Ra}=5 \times 10^{5}$. It can be observed that, for sufficiently low value of Planck number $\left(N_{\mathrm{CR}} \leq 0.5\right)$, both the radiation and convective heat transfer increase. The reason might be that, for low value of Planck number, radiation is assisted by natural convection. Looking up at (15) and (24), it becomes obvious that the contribution of radiation in total energy and total heat transfer should fade away in the limit $N_{\mathrm{CR}} \rightarrow$ $\infty$, and for sufficiently large $N_{\mathrm{CR}}$, the energy equation (15) gets decoupled from the radiant energy, whereas natural convection becomes the main mechanism to drive the flow. This is now evident from Figure 11 that, beyond $N_{\mathrm{CR}} 0.5$, there is a decrease in the radiation heat transfer with the increasing values of $N_{\mathrm{CR}}$, and it asymptotically reaches to zero in the limit $N_{\mathrm{CR}}>20$ in this case, whereas the convective heat transfer rate increases and approaches total heat transfer curve for $N_{\mathrm{CR}}>20$. Thus in this case for $N_{\mathrm{CR}}>20$, the overall heat transfer rate is represented by convective heat transfer rate and the contribution of radiative heat transfer vanishes. Therefore we can say that there is a smooth transition of flow from radiation dominated regime to convection dominated regime. This is also physically evident from the definition of Planck number that, for $N_{\mathrm{CR}} \gg 1$, the conduction effects would become dominant. However, the convective and total heat transfer attain almost a steady value beyond $N_{\mathrm{CR}}>15$, due to the fact that the contribution of radiation in the total thermal energy becomes less and less as we increase the value of $N_{\mathrm{CR}}$.

\section{Conclusion}

An investigation of the effect of radiation and natural convection of viscous incompressible fluid in a square open cavity

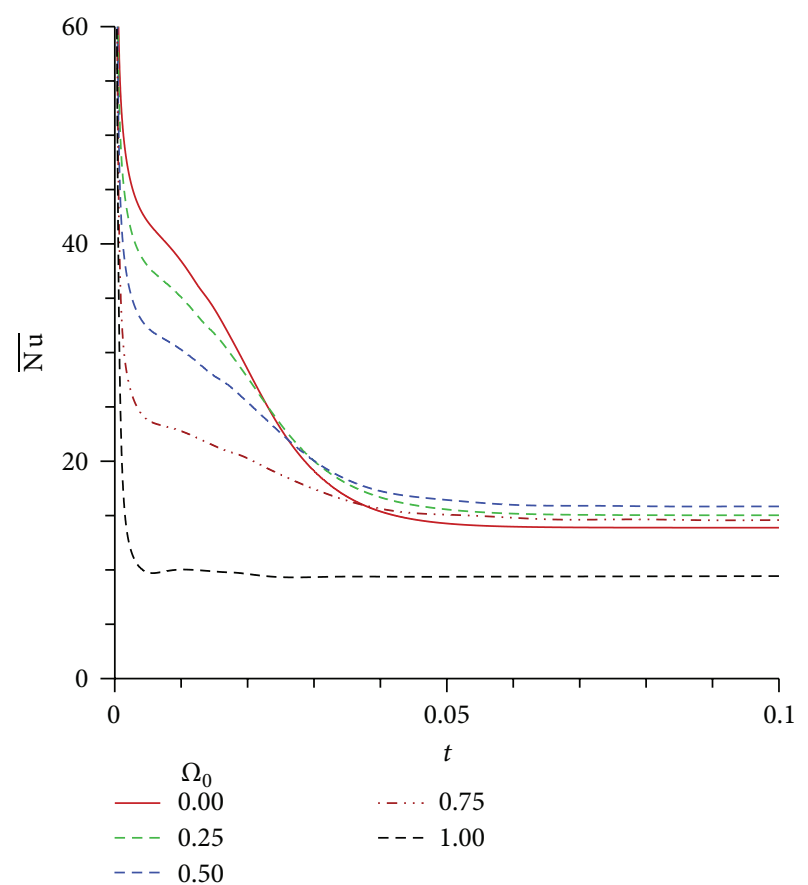

FIGURE 8: Average heat transfer rate against time while $\mathrm{Ra}=2 \times 10^{5}$, $\tau_{0}=0.5$, and $N_{\mathrm{CR}}=0.2$ for different values of scattering albedo $\Omega_{0}$.

has been carried out. The main focus was the study of flow pattern and heat transfer rates for different values of radiation parameters $0 \leq \tau_{0} \leq 2.5,0 \leq \Omega_{0} \leq 1$, and $0.05 \leq N_{\mathrm{CR}} \leq 20$. The following conclusions are obtained.

(1) It was seen that with the increase in the optical thickness the strength of flow and energy level of the fluid increases, which ultimately results in the negation of heat transfer rate. Convective heat transfer $\mathrm{Nu}_{C}$ decreases asymptotically to zero, whereas radiative heat transfer $\mathrm{Nu}_{R}$ first increases in the range $0 \leq \tau_{0} \leq$ 0.5 and then decreases due to rapid attenuation along propagation in the optically thick media.

(2) The total heat transfer is not significantly affected in the range $0 \leq \Omega_{0} \leq 0.75$, whereas due to rapid decrease in radiative heat transfer, overall heat transfer decreases near $\Omega_{0}=1$.

(3) Both the strength of flow and energy level of the fluid decrease with the increase in Planck number $N_{\mathrm{CR}}$. The contribution of radiative heat transfer asymptotically fades to zero with the increase in the value of $N_{\mathrm{CR}}$, whereas convective heat transfer remains the main mechanism of heat transfer for large values of $N_{\mathrm{CR}}$.

\section{Nomenclature}

\section{English Letters}

A: Aspect ratio

$C_{p}$ : Molar specific heat at constant pressure $\left(\mathrm{JK}^{-1}\right)$

$g:$ Acceleration due to gravity $\left(\mathrm{ms}^{-2}\right)$

$\bar{G}$ : Radiant energy $\mathrm{Wm}^{-2}$ 


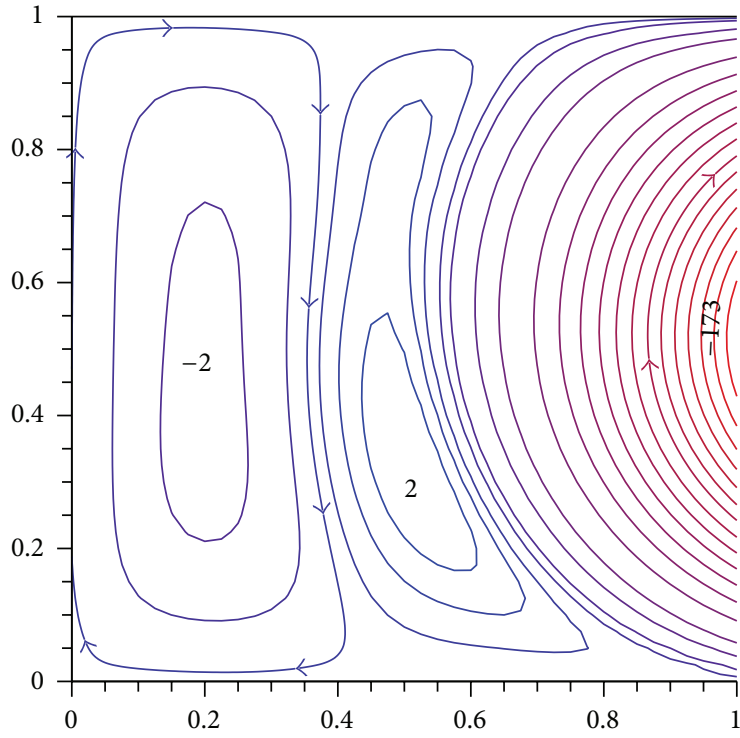

(a)

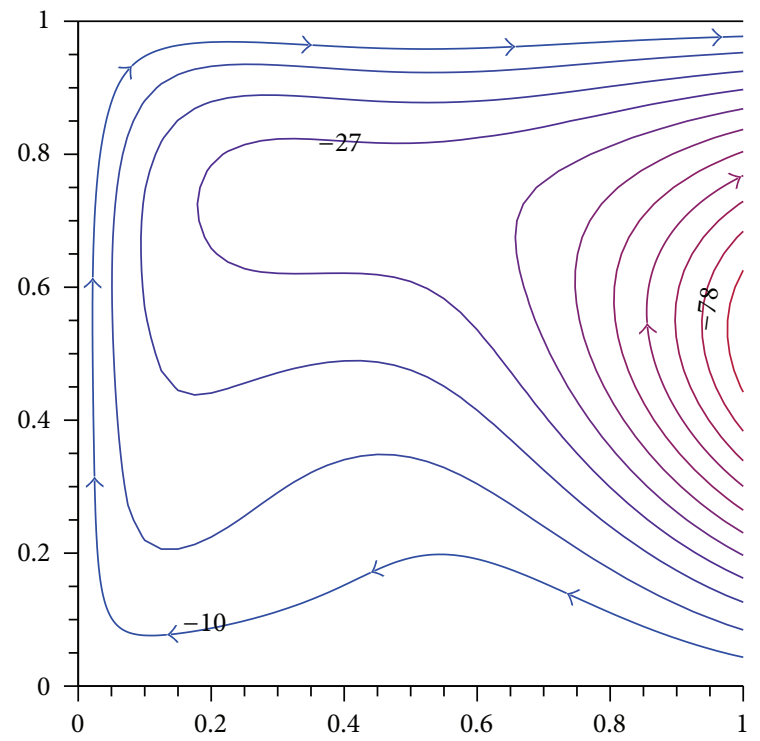

(b)

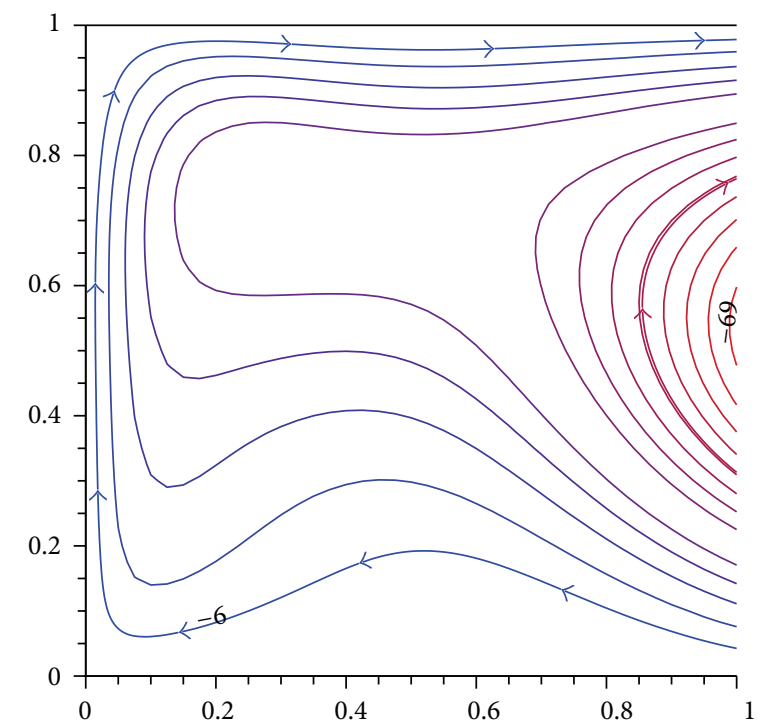

(c)

FIGURE 9: Streamlines for $\mathrm{Ra}=5 \times 10^{5}, \Omega_{0}=0.2$, and $\tau_{0}=1.0$ at (a) $N_{\mathrm{CR}}=0.2$, (b) $N_{\mathrm{CR}}=2.0$, and (c) $N_{\mathrm{CR}}=4.0$.

G: $\quad$ Dimensionless radiant energy

$\bar{H}$ : Heat function $\mathrm{Wm}^{-1}$

$H$ : Dimensionless heat function

$h$ : $\quad$ Mesh spacing

$(i, j)$ : Nodal locations of $(x, y)$ on grid

$I, J$ : The number of grid points in each direction

$k$ : Coefficient of thermal conductivity $\left(\mathrm{Wm}^{-1} \mathrm{~K}^{-1}\right)$

$N_{\mathrm{CR}}$ : Planck number

$\mathrm{Nu}_{C}$ : Convective Nusselt number

$\overline{\mathrm{Nu}}_{C}$ : Average convection Nusselt number

$\mathrm{Nu}_{R}$ : Radiation heat transfer

$\overline{\mathrm{Nu}}_{R}$ : Average radiation heat transfer

Nu: Total Nusselt number

$\overline{\mathrm{Nu}}$ : Average Nusselt numbers $\bar{p}: \quad$ Fluid pressure $(\mathrm{Pa})$

$\bar{q}_{r}: \quad$ Radiant flux $\mathrm{Wm}^{-2}$

$q_{r}: \quad$ Dimensionless radiant flux

Pr: $\quad$ Prandtl number

Ra: Raleigh number

$\bar{T}: \quad$ Dimensional temperature $(\mathrm{K})$

$\bar{T}_{H}, \bar{T}_{C}:$ Maximum and minimum temperature $(\mathrm{K})$

$\bar{T}_{0}: \quad$ Average/reference temperature $(\mathrm{K})$

$\bar{t}: \quad$ Dimensional time (s)

$t$ : $\quad$ Nondimensional time

$\bar{u}, \bar{v}: \quad$ Velocity components $\left(\mathrm{ms}^{-1}\right)$

$u, v: \quad$ Nondimensional velocity components

$X, Y: \quad$ Length and height of the cavity $(\mathrm{m})$

$\bar{x}, \bar{y}: \quad$ Dimensional coordinate axis (m)

$x, y$ : Nondimensional coordinate axis. 


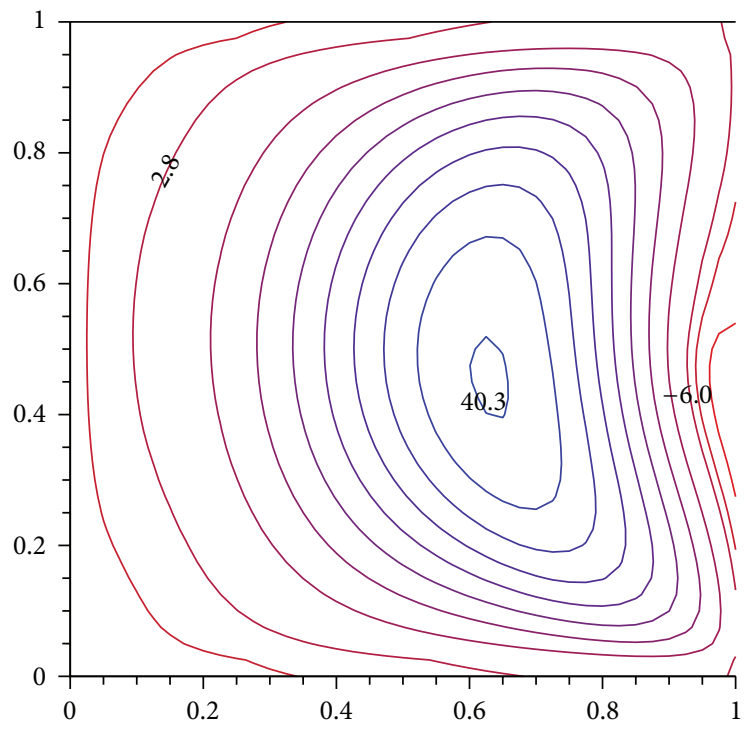

(a)

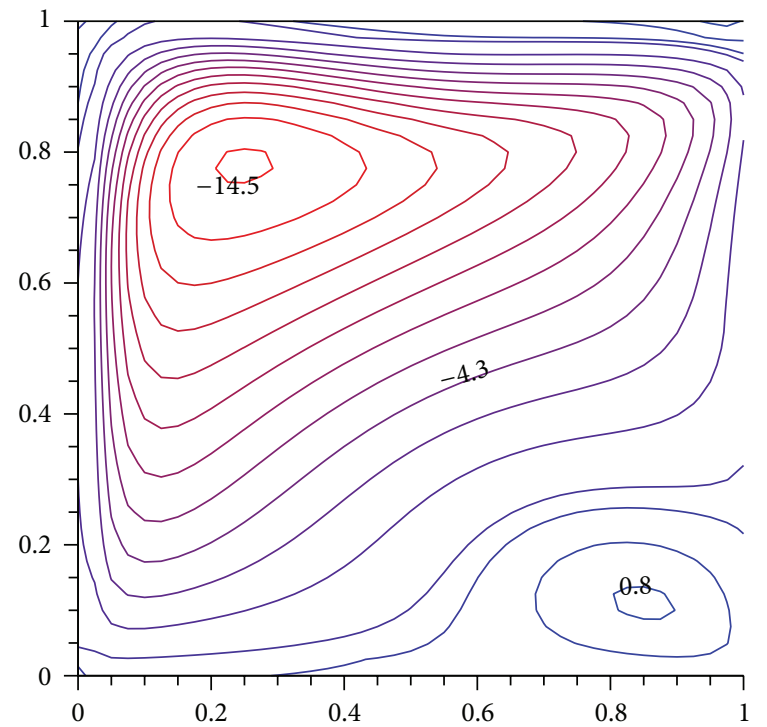

(b)

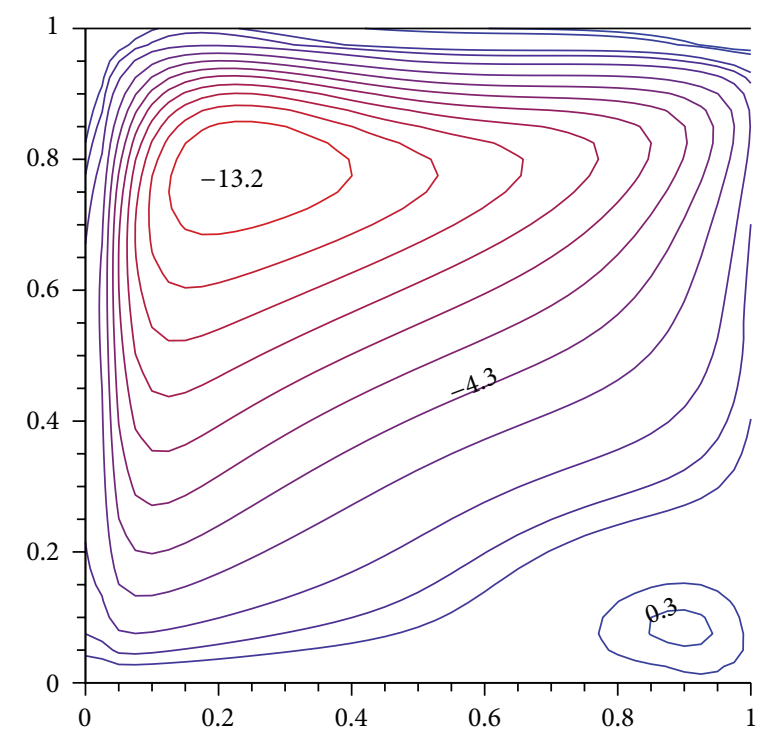

(c)

Figure 10: Heatlines for Ra $=5 \times 10^{5}, \Omega_{0}=0.2$, and $\tau_{0}=1.0$ at (a) $N_{\mathrm{CR}}=0.2$, (b) $N_{\mathrm{CR}}=2.0$, and (c) $N_{\mathrm{CR}}=4.0$.

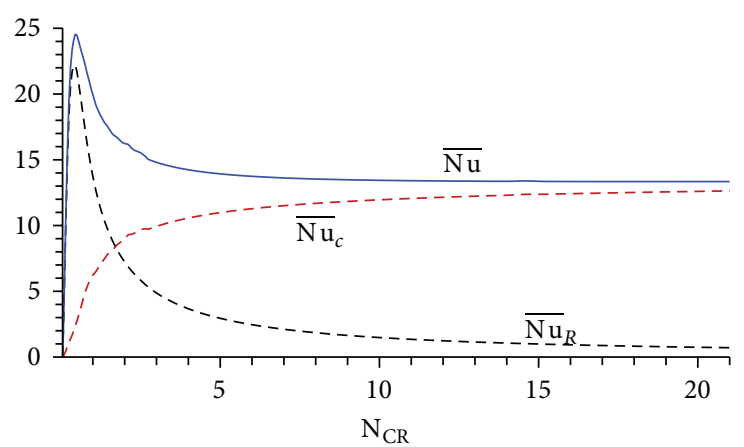

FIGURE 11: Heat transfer rate as a function of $N_{\mathrm{CR}}$ at $\mathrm{Ra}=5 \times 10^{5}, \Omega_{0}=0.2$, and $\tau_{0}=1.0$. 


\section{Greek Letters}

$\alpha:$ Thermal diffusivity $\left(k / \rho C_{p}\right)$
$\alpha_{m}:$ Mean extinction coefficient
$\beta:$ Thermal expansion coefficient $\left(\mathrm{K}^{-1}\right)$
$\epsilon_{i}:$ Wall emissivity/absorption
$\theta:$ Nondimensional temperature
$\mu: \quad$ Dynamic viscosity $\left(\mathrm{m}^{-1} \mathrm{~s}^{-1}\right)$
$\nu: \quad$ Kinematic viscosity $\mathrm{m}^{2} \mathrm{~s}^{-1} \mathrm{Kg}^{-1}$
$\rho:$ Density of fluid $\left(\mathrm{Kgm}^{-3}\right)$
$\sigma:$ Stefan-Boltzmann constant $\mathrm{Wm}^{-2} \mathrm{~K}^{-4}$
$\tau_{0}:$ Optical thickness
$\bar{\psi}:$ Stream function $\left(\mathrm{m}^{2} \mathrm{~s}^{-1} \mathrm{Kg}^{-1}\right)$
$\psi:$ Nondimensional stream function
$\Omega_{0}:$ Scattering Albedo
$\bar{\omega}:$ Dimensional vorticity function $\left(\mathrm{s}^{-1}\right)$
$\omega:$ Nondimensional vorticity function.

\section{Conflict of Interests}

The authors declare that there is no conflict of interests regarding the publication of this paper.

\section{References}

[1] R. Siegel and J. R. Howell, Thermal Radiation Heat Transfer, Hemisphere, Washington, Wash, USA, 3rd edition, 1992.

[2] M. F. Modest, Radiative Heat Transfer, Academic Press, New York, NY, USA, 2nd edition, 2003.

[3] D. W. Larson and R. Viskanta, "Transient combined laminar free convection and radiation in a rectangular enclosure," Journal of Fluid Mechanics, vol. 78, no. 1, pp. 65-85, 1976.

[4] G. Lauriat, "Gray fluids enclosed in vertical cavities," Journal of Heat Transfer, vol. 104, no. 4, pp. 609-615, 1982.

[5] C. Bouallou and J. F. Sacadura, "Thermal radiation, convection, and conduction in porous media contained in two-dimensional vertical cavities," Journal of Heat Transfer, vol. 113, no. 1, pp. 255258, 1991.

[6] A. Draoui, F. Allard, and C. Beghein, "Numerical analysis of heat transfer by natural convection and radiation in participating fluids enclosed in square cavities," Numerical Heat Transfer A: Applications, vol. 20, no. 2, pp. 253-261, 1991.

[7] A. G. Salinger, S. Brandon, R. Aris, and J. J. Derby, "Buoyancydriven flows of a radiatively participating fluid in a vertical cylinder heated from below," Proceedings Royal Society of London A, vol. 442, no. 1915, pp. 313-341, 1993.

[8] J. J. Derby, S. Brandon, and A. G. Salinger, "The diffusion and $P_{1}$ approximations for modeling buoyant flow of an optically thick fluid," International Journal of Heat and Mass Transfer, vol. 41, no. 11, pp. 1405-1415, 1998.

[9] E. H. Ridouane, M. Hasnaoui, and A. Campo, "Effects of surface radiation on natural convection in a rayleigh-benard square enclosure: steady and unsteady conditions," Heat and Mass Transfer/Waerme- und Stoffuebertragung, vol. 42, no. 3, pp. 214225, 2006.

[10] S. Shateyi, S. S. Motsa, and P. Sibanda, "The effects of thermal radiation, hall currents, soret, and dufour on MHD flow by mixed convection over a vertical surface in porous media," Mathematical Problems in Engineering, vol. 2010, Article ID 627475, 20 pages, 2010.
[11] M. M. Molla, S. C. Saha, and M. A. Hossain, "Radiation effect on free convection laminar flow along a vertical flat plate with streamwise sinusoidal surface temperature," Mathematical and Computer Modelling, vol. 53, no. 5-6, pp. 1310-1319, 2011.

[12] S. Siddiqa, M. A. Hossain, and S. C. Saha, "Natural convection flow with surface radiation along a vertical wavy surface," Numerical Heat Transfer A: Applications, vol. 64, no. 5, pp. 400415, 2013.

[13] R. C. Aziz, I. Hashim, and S. Abbasbandy, "Effects of thermocapillarity and thermal radiation on flow and heat transfer in a thin liquid film on an unsteady stretching sheet," Mathematical Problems in Engineering, vol. 2012, Article ID 127320, 14 pages, 2012.

[14] R. Nandkeolyar, M. Das, and P. Sibanda, "Unsteady hydromagnetic heat and mass transfer flow of a heat radiating and chemically reactive fluid past a flat porous plate with ramped wall temperature," Mathematical Problems in Engineering, vol. 2013, Article ID 381806, 12 pages, 2013.

[15] X. Liu, G. Gong, and H. Cheng, "Combined natural convection and radiation heat transfer of various absorbing-emittingscattering media in a square cavity," Advances in Mechanical Engineering, vol. 2014, Article ID 403690, 10 pages, 2014.

[16] S. Kimura and A. Bejan, "The "Heatline" visualization of convective heat transfer," ASME Journal of Heat Transfer, vol. 105, no. 4, pp. 916-919, 1983.

[17] F. L. Bello-Ochende, "A heat function formulation for thermal convection in a square cavity," International Communications in Heat and Mass Transfer, vol. 15, no. 2, pp. 193-202, 1988.

[18] V. A. F. Costa, "Unification of the streamline, heatline and massline methods for the visualization of two-dimensional transport phenomena," International Journal of Heat and Mass Transfer, vol. 42, no. 1, pp. 27-33, 1999.

[19] V. A. F. Costa, "Heatline and massline visualization of laminar natural convection boundary layers near a vertical wall," International Journal of Heat and Mass Transfer, vol. 43, no. 20, pp. 3765-3774, 2000.

[20] Q. H. Deng and G. F. Tang, "Numerical visualization of mass and heat transport for mixed convective heat transfer by streamline and heatline," International Journal of Heat and Mass Transfer, vol. 45, no. 11, pp. 2387-2396, 2002.

[21] M. A. Hossain, M. Saleem, and R. S. R. Gorla, "Surfaceradiation effect on natural convection flow in a fluid-saturated non-Darcy porous medium enclosed by non-isothermal walls," International Journal of Numerical Methods for Heat \& Fluid Flow, vol. 23, no. 8, pp. 1320-1339, 2013.

[22] M. Saleem, S. Asghar, and M. A. Hossain, "Natural convection flow in an open rectangular cavity with cold sidewalls and constant volumetric heat source," Proceedings of the Institution of Mechanical Engineers C: Journal of Mechanical Engineering Science, vol. 225, no. 5, pp. 1191-1201, 2011.

[23] M. Saleem, M. A. Hossain, S. Mahmud, and I. Pop, "Entropy generation in Marangoni convection flow of heated fluid in an open ended cavity," International Journal of Heat and Mass Transfer, vol. 54, no. 21-22, pp. 4473-4484, 2011.

[24] J. F. Hinojosa, R. E. Cabanillas, G. Alvarez, and C. E. Estrada, "Nusselt number for the natural convection and surface thermal radiation in a square tilted open cavity," International Communications in Heat and Mass Transfer, vol. 32, no. 9, pp. 1184-1192, 2005.

[25] H. Nouanegue, A. Muftuoglu, and E. Bilgen, "Conjugate heat transfer by natural convection, conduction and radiation in 
open cavities," International Journal of Heat and Mass Transfer, vol. 51, no. 25-26, pp. 6054-6062, 2008.

[26] E. M. Sparrow and J. P. Abraham, "A new buoyancy model replacing the standard pseudo-density difference for internal natural convection in gases," International Journal of Heat and Mass Transfer, vol. 46, no. 19, pp. 3583-3591, 2003.

[27] P. J. Roache, Computational Fluid Dynamics, Hermosa, Albuquerque, NM, USA, 2nd edition, 1988. 


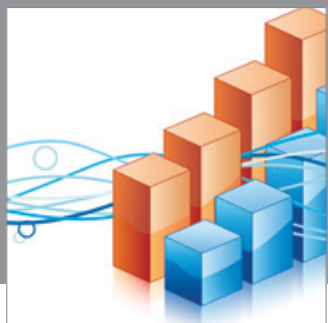

Advances in

Operations Research

mansans

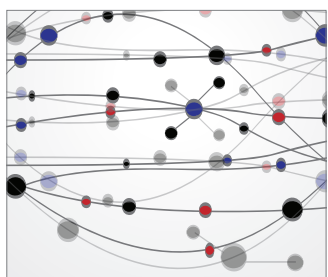

The Scientific World Journal
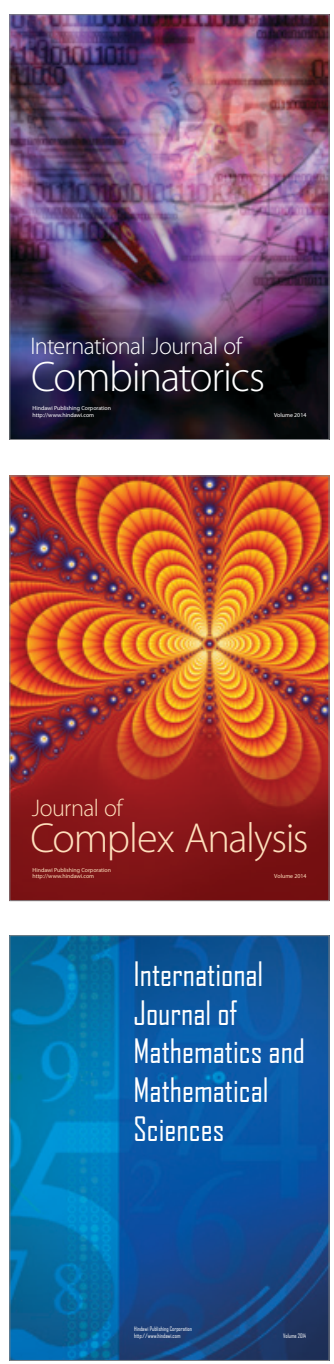
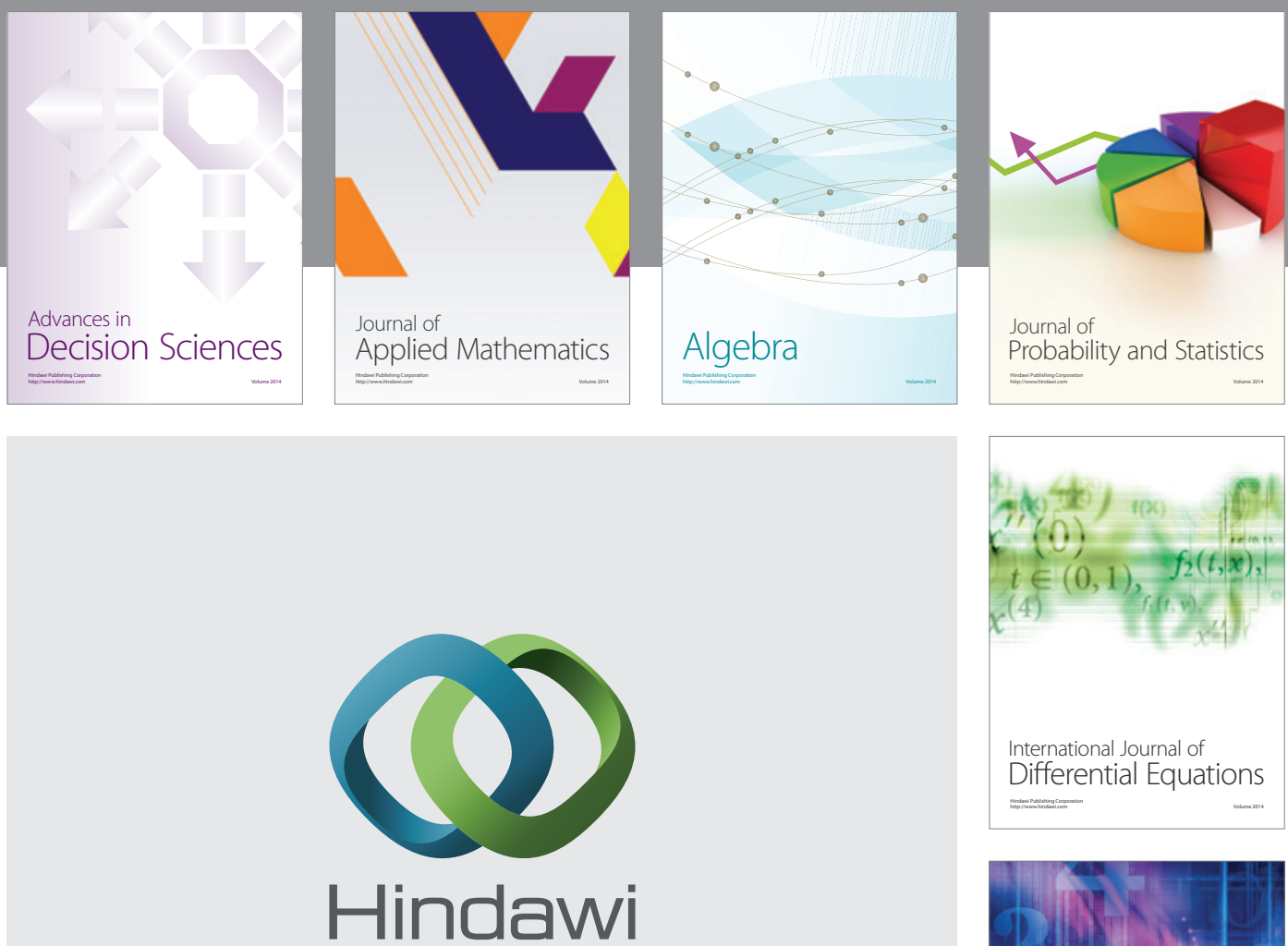

Submit your manuscripts at http://www.hindawi.com
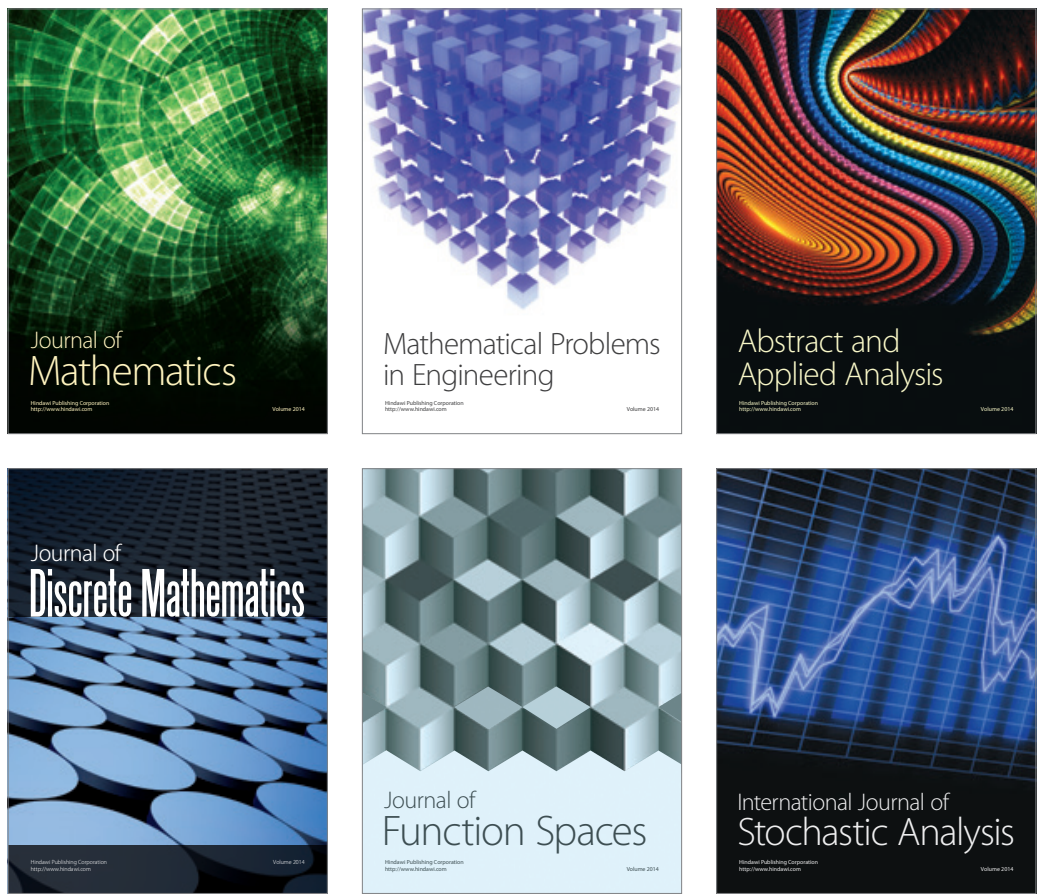

Journal of

Function Spaces

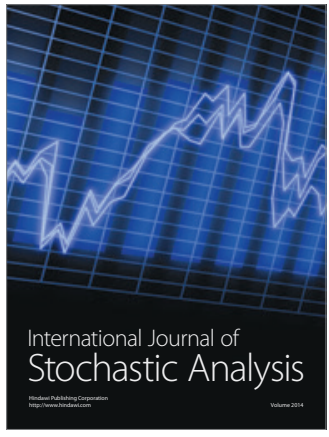

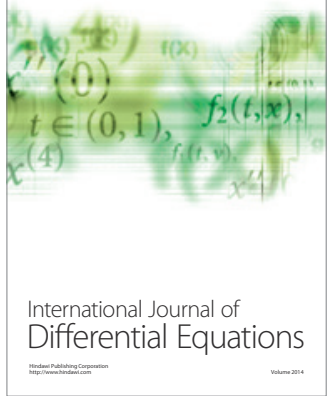
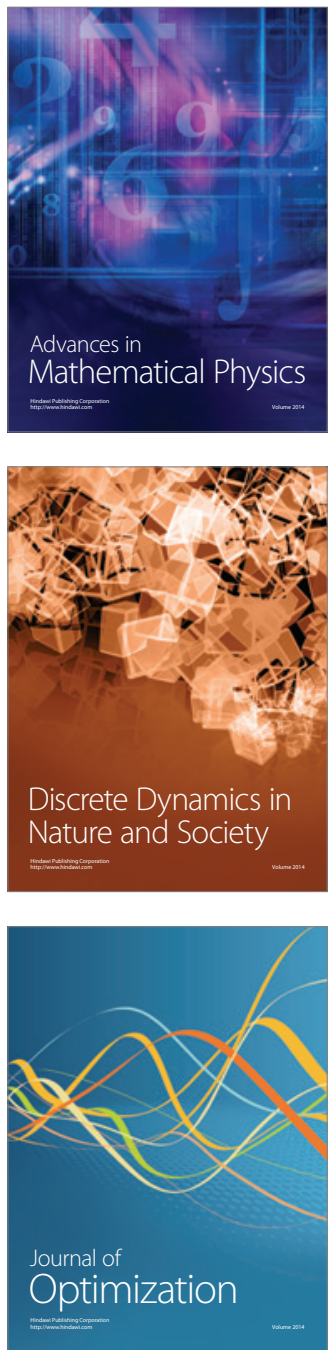